PROCEEDINGS OF THE

AMERICAN MATHEMATICAL SOCIETY

Volume 131, Number 7, Pages 2063-2070

S 0002-9939(03)07001-1

Article electronically published on February 5, 2003

\title{
RENORMING OF $C(K)$ SPACES
}

\author{
JAN RYCHTÁŘ
}

(Communicated by Jonathan M. Borwein)

\begin{abstract}
If $K$ is a scattered Eberlein compact space, then $C(K)^{*}$ admits an equivalent dual norm that is uniformly rotund in every direction. The same is shown for the dual to the Johnson-Lindenstrauss space $\mathrm{JL}_{2}$.
\end{abstract}

\section{INTRODUCTION}

We will find classes of Banach spaces whose duals admit equivalent dual norms that are uniformly rotund in every direction (URED) or pointwise uniformly rotund (p-UR) (definitions are given below). The notion of p-UR covers both the weak and weak* uniform rotundity ( $\mathrm{W}^{*} \mathrm{UR}$ ). It can be shown from the Šmulyan theorem (see, e.g., [5 p. 63]), that the existence of a dual p-UR norm on $X^{*}$ implies the existence of a "big" set in $X^{* *}$ on which the bidual norm is uniformly Gâteaux differentiable.

In Section 2 we will prove a three-space-like theorem for the following properties of a Banach space $X: X^{*}$ admits an equivalent dual URED $(p-U R)$ norm. This result enables us to renorm duals to spaces, such as the Johnson-Lindenstrauss space or $C(K)$ for $K$ scattered with $K^{(\omega)}=\emptyset$, by dual norms that are simultaneously locally uniformly rotund (LUR) and p-UR. On the example of $C(K)$, where $K$ is the so-called "two arrow" compact space, it is shown that properties of the duals to be equivalently renormed by dual URED norm (or p-UR norm) are not three space properties.

In Section 3, we will apply previous results, use a result from [1] and a method from [9, 11. It will be proved that if $K$ is an Eberlein and scattered compact space, then $C(K)^{*}$ admits an equivalent dual LUR and p-UR norm.

Recently it was shown in 6] that if $X^{*}$ admits weak* uniformly rotund norm, then $X$ is a subspace of weakly compactly generated space. However, in 12, Th. $1]$ it is shown that if $X$ has an unconditional Schauder basis and $X^{*}$ admits an equivalent (not necessarily dual) URED norm, then $X^{*}$ admits an equivalent dual weak ${ }^{*}$ uniformly rotund norm. Hence the space $\mathrm{JL}_{2}$ from Section 2 shows that Theorem 1 in [12] does not hold without the assumption of unconditional Schauder

Received by the editors July 15, 2001.

2000 Mathematics Subject Classification. Primary 46B03, 46E10.

Key words and phrases. Eberlein compacts, uniform rotundity in every direction.

Supported in part by GAČR 201/01/1198, A 1019003, NSERC 7926 and GAUK 277/2001. This paper is based on part of the author's Ph.D. thesis written under the supervision of Professor V. Zizler. 
basis. From the result in Section 3 we can deduce that if $K$ is scattered Eberlein compact, that is not uniform Eberlein compact, then $C(K)^{*}$ is a dual to the weakly compactly generated space and admits an equivalent dual p-UR norm, but no equivalent dual weak* uniformly rotund norm, i.e., there is weakly compactly generated Banach space $X$, such that its dual $X^{*}$ admits an equivalent dual URED and LUR norm and no $\mathrm{W}^{*} \mathrm{UR}$ norm.

Let $(X,\|\|$.$) be a Banach space. Let S_{X}$ and $B_{X}$ denote the unit sphere and the unit ball respectively, i.e., $S_{X}=\{x \in X ;\|x\|=1\}$ and $B_{X}=\{x \in X ;\|x\| \leq 1\}$. The norm $\|$.$\| on a Banach space X$ is said to be uniformly rotund in every direction (URED for short), if $\lim _{n \rightarrow \infty}\left\|x_{n}-y_{n}\right\|=0$ whenever $x_{n}, y_{n} \in S_{X}$ are such that $x_{n}-y_{n}=\lambda_{n} z$ for some $z \in X, \lambda_{n} \in \mathbb{R}$ and $\lim _{n \rightarrow \infty}\left\|x_{n}+y_{n}\right\|=2$. We will say that the norm $\|\cdot\|$ on $X$ is pointwise uniformly rotund $(p-U R)$, if there exists a $w^{*}$-dense set $\mathbb{F} \subset X^{*}$ such that $\lim _{n \rightarrow \infty} f\left(x_{n}-y_{n}\right)=0$ whenever $x_{n}, y_{n} \in S_{(X,\|.\|)}$, $\lim _{n \rightarrow \infty}\left\|x_{n}+y_{n}\right\|=2$, and $f \in \mathbb{F}$. More precisely, we say that the norm is $p$-UR with $\mathbb{F}$. Clearly, if the norm is p-UR, then it is URED. In the case of a dual Banach space $X=Y^{*}$ we say that the norm is weak* uniformly rotund ( $W^{*} U R$ ), if it is pUR with $\mathbb{F}=Y \subset Y^{* *}$. The norm $\|$.$\| is said to be locally uniformly rotund (L U R)$, if $\lim _{n \rightarrow \infty}\left\|x-x_{n}\right\|=0$ whenever $x, x_{n} \in S_{X}$ are such that $\lim _{n \rightarrow \infty}\left\|x+x_{n}\right\|=2$.

A compact space $K$ is an Eberlein compact if $K$ is homeomorphic to a weakly compact subset of a Banach space in its weak topology. A compact space $K$ is a uniform Eberlein compact if $K$ is homeomorphic to a weakly compact subset of a Hilbert space. A compact space is called scattered if every closed subset $L \subset K$ has an isolated point in $L$. For scattered compact spaces the Cantor derivative sets are defined as follows: $K^{(0)}=K, K^{(1)}=K^{\prime}$ is the set of all limit points of $K$. If $\alpha$ is an ordinal and $K^{(\beta)}$ are defined for all $\beta<\alpha$, then we put $K^{(\alpha)}=\left(K^{(\beta)}\right)^{\prime}$ for $\alpha=\beta+1$ and $K^{(\alpha)}=\bigcap_{\beta<\alpha} K^{(\beta)}$ for $\alpha$ a limit ordinal.

If we consider spaces such as $c_{0}(\Gamma), \ell_{1}(\Gamma), l_{\infty}(\Gamma)$, by the symbol $e_{\gamma}$ we mean the standard unit vector.

For more information in this area we refer to [3], [5], [7, Ch. 12], 10] and [14].

\section{The THREE SPACE PROBLEM}

Theorem 1. Let $X$ be a Banach space such that $c_{0}(\Gamma) \subset X$. Let the dual to $Y=X / c_{0}(\Gamma)$ admit an equivalent dual $p$-UR (URED) norm. Then $X^{*}$ admits an equivalent dual p-UR (URED) norm.

Proof. Let $i: c_{0}(\Gamma) \rightarrow X$ be the inclusion map and $q: X \rightarrow Y$ be the quotient map. Then the dual mappings are $i^{*}: X^{*} \rightarrow c_{0}(\Gamma)^{*}$, which is a quotient map and a restriction, and $q^{*}: Y^{*} \rightarrow X^{*}$, which is an inclusion. Because of the lifting property of the space $\ell_{1}(\Gamma) \cong c_{0}(\Gamma)^{*}$ there is a bounded linear map $l: \ell_{1}(\Gamma) \rightarrow X^{*}$ (the so-called lifting; see, e.g., [4]) such that $i^{*}(l(e))=e$ for all $e \in \ell_{1}(\Gamma)$. Hence we have an isomorphism $X^{*} \cong \ell_{1}(\Gamma) \oplus Y^{*}$, where the duality between $(f, g) \in \ell_{1}(\Gamma) \oplus Y^{*}$ and $x \in X$ is given by the formula

$$
\langle(f, g), x\rangle=\langle l(f), x\rangle+\left\langle q^{*}(g), x\right\rangle .
$$

Let $\|.\|_{Y^{*}}$ be a dual norm on $Y^{*}$ which is p-UR with $\mathbb{F}$. We will prove that there is an equivalent dual norm $\|\cdot\|_{u}$ on $X^{*}$, that is, p-UR with $\mathbb{G}=\left\{\left(e_{\gamma}, 0\right) ; \gamma \in\right.$ $\Gamma\} \cup\{(0, f) ; f \in \mathbb{F}\}$, where we identify $X^{* *}$ with $l_{\infty}(\Gamma) \oplus Y^{* *}$ and where $\left\{e_{\gamma} ; \gamma \in \Gamma\right\}$ denote the standard unit vectors in $c_{0}(\Gamma) \subset l_{\infty}(\Gamma)$. The proof that the norm $\|\cdot\|_{u}$ is URED if $\|\cdot\|_{Y^{*}}$ is URED proceeds in the same way. 
Let $\|\cdot\|_{X^{*}}$ be a dual norm on $X^{*}$ which extends the norm $\|\cdot\|_{Y^{*}}$. Let $\|\cdot\|_{\ell_{1}(\Gamma)}$ be the standard norm on $\ell_{1}(\Gamma)$. We choose $a>1$ such that

$$
a^{-1}\|(f, g)\|_{X^{*}} \leq\|f\|_{\ell_{1}(\Gamma)}+\|g\|_{Y^{*}} \leq a\|(f, g)\|_{X^{*}} .
$$

Put

$$
\|(f, g)\|_{w}=\left(\|f\|_{\ell_{1}(\Gamma)}^{2}+\|f\|_{l_{2}(\Gamma)}^{2}+\|g\|_{Y^{*}}^{2}\right)^{\frac{1}{2}} .
$$

This is an equivalent norm on $X^{*} \cong \ell_{1}(\Gamma) \oplus Y^{*}$. The norm $\|\cdot\|_{w}$ need not be a dual norm, but it is p-UR with $\mathbb{G}$. This convexity property will be used at the end of this proof. To have a dual norm, let us define

$$
\|(f, g)\|=\|(f, g)\|_{w}+a\|f\|_{\ell_{1}(\Gamma)} .
$$

Observation. The norm $\|$.$\| is a dual norm on X^{*}$.

Proof of the Observation. We will follow the proof published in [8] and show that the unit ball is closed in the weak* topology. To prove this, let $\left\{\left(f_{\alpha}, g_{\alpha}\right)\right\}_{\alpha \in A}$ be a net in the unit ball in $\left(X^{*},\|\|.\right)$, which weak* converges to $(f, g)$. Because $c_{0}(\Gamma) \subset X$ and $\ell_{1}(\Gamma) \cong c_{0}(\Gamma)^{*},\left\{f_{\alpha}\right\}_{\alpha \in A}$ converges coordinatewise to $f$. To see this, choose $x \in c_{0}(\Gamma)$. We have

$$
\begin{aligned}
\left\langle\left(f_{\alpha}, g_{\alpha}\right), i(x)\right\rangle & =\left\langle l\left(f_{\alpha}\right), i(x)\right\rangle+\left\langle q^{*}\left(g_{\alpha}\right), i(x)\right\rangle \\
& =\left\langle i^{*}\left(l\left(f_{\alpha}\right)\right), x\right\rangle+\left\langle g_{\alpha}, q(i(x))\right\rangle=\left\langle f_{\alpha}, x\right\rangle .
\end{aligned}
$$

To estimate the norm of $(f, g)$ we will decompose $f_{\alpha}$ in a special way. For each $\alpha \in A$, we can find elements $f_{\alpha}^{1}, f_{\alpha}^{2} \in \ell_{1}(\Gamma)$ such that $f_{\alpha}=f_{\alpha}^{1}+f_{\alpha}^{2}$, the supports of $f_{\alpha}^{1}, f_{\alpha}^{2}$ are disjoint and $\lim _{\alpha \in A}\left\|f_{\alpha}-f_{\alpha}^{1}\right\|_{\ell_{1}(\Gamma)}=0$. By passing to a subnet, we can

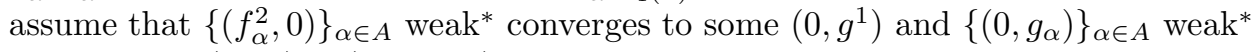
converges to $\left(0, g_{2}\right)=\left(0, g-g_{1}\right)$. Then

$$
\begin{gathered}
\|f\|_{\ell_{1}(\Gamma)} \leq \liminf _{\alpha \in A}\left\|f_{\alpha}^{1}\right\|_{\ell_{1}(\Gamma)}, \\
\left\|g_{1}\right\|_{Y^{*}}=\left\|\left(0, g_{1}\right)\right\|_{X^{*}} \leq \liminf _{\alpha \in A}\left\|\left(f_{\alpha}^{2}, 0\right)\right\|_{X^{*}} \leq a \liminf _{\alpha \in A}\left\|f_{\alpha}^{2}\right\|_{\ell_{1}(\Gamma)}, \\
\left\|g_{2}\right\|_{Y^{*}}=\left\|\left(0, g_{2}\right)\right\|_{X^{*}} \leq \liminf _{\alpha \in A}\left\|\left(0, g_{\alpha}\right)\right\|_{X^{*}}=\liminf _{\alpha \in A}\left\|g_{\alpha}\right\|_{Y^{*}},
\end{gathered}
$$

where we used that $\|.\|_{X^{*}}$ is the dual norm. It follows from previous estimates that

$$
\begin{aligned}
\|(f, g)\| & =a\|f\|_{\ell_{1}(\Gamma)}+\left(\|f\|_{\ell_{1}(\Gamma)}^{2}+\|f\|_{\ell_{2}(\Gamma)}^{2}+\left\|g_{1}+g_{2}\right\|_{Y^{*}}^{2}\right)^{\frac{1}{2}} \\
& \leq a\|f\|_{\ell_{1}(\Gamma)}+\left\|g_{1}\right\|_{Y^{*}}+\left(\|f\|_{\ell_{1}(\Gamma)}^{2}+\|f\|_{\ell_{2}(\Gamma)}^{2}+\left\|g_{2}\right\|_{Y^{*}}^{2}\right)^{\frac{1}{2}} \\
& \leq \liminf _{\alpha \in A}\left(a\left\|f_{\alpha}^{1}\right\|_{\ell_{1}(\Gamma)}+a\left\|f_{\alpha}^{2}\right\|_{\ell_{1}(\Gamma)}+\left(\left\|f_{\alpha}^{1}\right\|_{\ell_{1}(\Gamma)}^{2}+\left\|f_{\alpha}^{1}\right\|_{\ell_{2}(\Gamma)}^{2}+\left\|g_{\alpha}\right\|_{Y^{*}}^{2}\right)^{\frac{1}{2}}\right) \\
& \leq \limsup _{\alpha \in A}\left\|\left(f_{\alpha}, g_{\alpha}\right)\right\| \leq 1 .
\end{aligned}
$$

Thus dual unit ball is $w^{*}$-closed and the Observation is proved.

We will continue with the proof of Theorem 1 . Let us define the norm $\|\cdot\|_{u}$ on $X^{*}$ by the formula

$$
\|(f, g)\|_{u}^{2}=\|(f, g)\|^{2}+\|f\|_{\ell_{1}(\Gamma)}^{2} .
$$

It is a dual norm, because it is $w^{*}$-lower semicontinuous as is the seminorm $\|\cdot\|_{\ell_{1}(\Gamma)}$ on $X^{*}$. We prove that it is p-UR with $\mathbb{G}$. To do this, we use the following fact, which can be found with the proof in [5, Ch. II]. 
Fact. Let $a_{n}, b_{n}$ be bounded elements of a Banach space $(E,\|\|$.$) such that$

$$
\lim _{n \rightarrow \infty}\left(2\left\|a_{n}\right\|^{2}+2\left\|b_{n}\right\|^{2}-\left\|a_{n}+b_{n}\right\|^{2}\right)=0 .
$$

Then $\lim _{n \rightarrow \infty}\left(\left\|a_{n}\right\|-\left\|b_{n}\right\|\right)=0$ and $\lim _{n \rightarrow \infty}\left(\left\|a_{n}\right\|+\left\|b_{n}\right\|-\left\|a_{n}+b_{n}\right\|\right)=0$.

Now assume that $x_{n}=\left(x_{n}^{1}, x_{n}^{2}\right), y_{n}=\left(y_{n}^{1}, y_{n}^{2}\right) \in X^{*}$ satisfy

$$
\lim _{n \rightarrow \infty}\left(2\left\|x_{n}\right\|_{u}^{2}+2\left\|y_{n}\right\|_{u}^{2}-\left\|x_{n}+y_{n}\right\|_{u}^{2}\right)=0 .
$$

By the previous Fact we have

$$
\lim _{n \rightarrow \infty}\left(2\left\|x_{n}^{1}\right\|_{\ell_{1}(\Gamma)}^{2}+2\left\|y_{n}^{1}\right\|_{\ell_{1}(\Gamma)}^{2}-\left\|x_{n}^{1}+y_{n}^{1}\right\|_{\ell_{1}(\Gamma)}^{2}\right)=0 .
$$

And again by the Fact

$$
\begin{gathered}
\lim _{n \rightarrow \infty}\left(\left\|x_{n}^{1}\right\|_{\ell_{1}(\Gamma)}-\left\|y_{n}^{1}\right\|_{\ell_{1}(\Gamma)}\right)=0, \\
\lim _{n \rightarrow \infty}\left(\left\|x_{n}^{1}\right\|_{\ell_{1}(\Gamma)}+\left\|y_{n}^{1}\right\|_{\ell_{1}(\Gamma)}\right)-\lim _{n \rightarrow \infty}\left\|x_{n}^{1}+y_{n}^{1}\right\|_{\ell_{1}(\Gamma)}=0 .
\end{gathered}
$$

By (1) and by the Fact it follows that

$$
\lim _{n \rightarrow \infty}\left(2\left\|x_{n}\right\|^{2}+2\left\|y_{n}\right\|^{2}-\left\|x_{n}+y_{n}\right\|^{2}\right)=0 .
$$

Hence by the Fact

$$
\lim _{n \rightarrow \infty}\left(\left\|x_{n}\right\|+\left\|y_{n}\right\|\right)-\lim _{n \rightarrow \infty}\left\|x_{n}+y_{n}\right\|=0 .
$$

By (2) and (3) we have

$$
\begin{aligned}
\lim _{n \rightarrow \infty}\left(\left\|x_{n}\right\|_{w}+\left\|y_{n}\right\|_{w}\right) & =\lim _{n \rightarrow \infty}\left\|x_{n}+y_{n}\right\|_{w}, \\
\lim _{n \rightarrow \infty}\left\|x_{n}\right\|_{w} & =\lim _{n \rightarrow \infty}\left\|y_{n}\right\|_{w} .
\end{aligned}
$$

Hence

$$
\lim _{n \rightarrow \infty}\left\|\frac{x_{n}}{\left\|x_{n}\right\|_{w}}+\frac{y_{n}}{\left\|y_{n}\right\|_{w}}\right\|_{w}=\lim _{n \rightarrow \infty}\left\|\frac{x_{n}}{\left\|x_{n}\right\|_{w}}+\frac{y_{n}}{\left\|x_{n}\right\|_{w}}\right\|_{w}=2 .
$$

The norm $\|\cdot\|_{w}$ on $\ell_{1}(\Gamma) \oplus Y^{*}$ is p-UR with $\mathbb{G}$; thus, for all $G \in \mathbb{G}$ we have

$$
\lim _{n \rightarrow \infty} G\left(\frac{x_{n}}{\left\|x_{n}\right\|_{w}}-\frac{y_{n}}{\left\|x_{n}\right\|_{w}}\right)=0
$$

which finishes the proof of Theorem 1 .

Remark. Moreover, if the norm $\|\cdot\|_{Y^{*}}$ on $Y^{*}$ is LUR, the norm $\|\cdot\|_{u}$ on $X^{*}$ is LUR as well because the norm $\|\cdot\|_{w}$ is.

Corollary 2. Let $K$ be a scattered compact space with $K^{(\omega)}=\emptyset$. Then $C(K)^{*}$ admits an equivalent dual norm that is simultaneously LUR and $p$-UR with $\mathbb{F}=$ $\left\{e_{k} ; k \in K\right\} \subset c_{0}(K) \subset C(K)^{* *}$.

Proof. By a compactness argument, there is some $n \in \mathbb{N}$ such that $K^{(n)}=\emptyset$. We shall prove the corollary by an induction. If $n=0,1$, the claim is trivial. Let $n>1$. It is easy to see that the space $Y=\left\{f \in C(K) ;\left.f\right|_{K^{\prime}}=0\right\}$ is isometric to the space $c_{0}\left(K \backslash K^{\prime}\right)$. Moreover, $C(K) / Y=C\left(K^{\prime}\right)$, so we can use Theorem 1.

In fact, the following theorem holds.

Theorem (Deville). Let $K$ be a scattered compact space, such that $K^{\left(\omega_{1}\right)}=\emptyset$. Then $C(K)^{*}$ admits an equivalent dual norm, that is LUR and $p$-UR. 
Proof. See [5, Theorem 7.4.7]. There is an equivalent dual LUR norm constructed on $C(K)^{*}$. One can compute that this norm is, moreover, p-UR with $\mathbb{F}=\left\{e_{k} ; k \in\right.$ $K\}$.

Note that it is shown in [8] (see also [13]) that there is a Banach space $\mathrm{JL}_{2}$ with the following properties:

(1) $c_{0} \subset \mathrm{JL}_{2}, \mathrm{JL}_{2} / c_{0}=\ell_{2}(\Gamma)$, where the cardinality of the set $\Gamma$ is a continuum,

(2) $\mathrm{JL}_{2}$ is not a subspace of any WCG space; in particular, $\mathrm{JL}_{2}$ is not isomorphic to the $c_{0} \oplus \ell_{2}(\Gamma)$,

(3) there is an equivalent dual LUR norm on $\mathrm{JL}_{2}^{*}$.

From Theorem 1 we can obtain a stronger result.

Theorem 3. There is an equivalent dual norm on the space $J L^{*}$ that is LUR and $p$-UR with $\mathbb{F}$, where $\mathbb{F}$ is the canonical imbedding of $c_{0} \oplus \ell_{2}(\Gamma)$ into $\ell_{\infty} \oplus \ell_{2}(\Gamma) \cong J L_{2}^{* *}$.

It is shown in [5. pp. 299-305], that if $K$ is a so-called "two arrow" compact space, then $C([0,1]) \subset C(K), C(K) / C([0,1])=c_{0}([0,1])$ and $C(K)$ has no equivalent Gâteaux smooth norm. It means that there is no dual equivalent strictly convex norm on $C(K)^{*}$. It means that $C(K)^{*}$ does not admit an equivalent dual URED norm, although both $C([0,1])^{*}$ and $c_{0}([0,1])^{*}$ do admit an equivalent dual p-UR norms.

\section{Scattered Eberlein COMPaCt SPACES}

Theorem 4. Let $K$ be a scattered compact space such that $K=\bigcup_{n=1}^{\infty} K_{n}$, and for all $n \in \mathbb{N}$ let $C\left(K_{n}\right)^{*}$ admit an equivalent dual $p$-UR norm with $\mathbb{F}_{n}=\left\{e_{k} ; k \in K_{n}\right\}$. Then $C(K)^{*}$ admits an equivalent dual $p$-UR norm with $\mathbb{F}=\left\{e_{k} ; k \in K\right\}$.

Proof. This proof is similar to the proof of Theorem 2.7.16 in [5], which states, that the space $L_{1}(\Omega)$ admits a norm that is LUR and URED.

As in [9], we can define the operator $T: C(K) \rightarrow \sum_{\ell_{2}} C\left(K_{n}\right)$ by the formula $T(f)=\left(\left.\frac{1}{n^{2}} f\right|_{K_{n}}\right)$. For $k \in K$ put $N(k)=\left\{n \in \mathbb{N} ; k \in K_{n}\right\}$. For $k \in K, n \in N(k)$ let $\widetilde{k}_{n}$ denote a copy of $k$ in $K_{n}$. For $A \subset K$ put $\widetilde{A}=\left\{\widetilde{k}_{n} ; k \in A, n \in N(k)\right\}$. By Rudin's Theorem (see [7]) $C(K)^{*}$ is isometric to the space $l_{1}(K)$ and the canonical norm $\|\cdot\|_{1}$ is a dual norm on $C(K)^{*}$, the same holds for $K_{n}$ 's. Without loss of generality, we can assume, that the p-UR norms are uniformly close to the original norms on $C\left(K_{n}\right)^{*}$. Hence $\left(\sum_{\ell_{2}} C\left(K_{n}\right)\right)^{*} \cong \sum_{\ell_{2}} \ell_{1}\left(K_{n}\right)$ and $\left(\sum_{\ell_{2}} C\left(K_{n}\right)\right)^{*}$ admits an equivalent dual norm $\|\cdot\|_{\Sigma}$, which is p-UR with $\mathbb{G}=\left\{e_{\tilde{k}_{n}} ; k \in K, n \in N(k)\right\}$. The dual operator $T^{*}:\left(\sum_{\ell_{2}} C\left(K_{n}\right)\right)^{*} \rightarrow C(K)^{*}$ is given by

$$
T^{*}\left(y^{*}\right)=\left(\sum_{n \in N(k)} \frac{1}{n^{2}} y^{*}\left(\widetilde{k}_{n}\right)\right)_{k \in K} .
$$

The range of $T^{*}$ is a dense set in $C(K)^{*}$. Now, we shall use the standard LUR renorming method. For $n \in \mathbb{N}$ and $x \in \ell_{1}(K)$ we define

$$
\begin{gathered}
|x|_{n}^{2}=\inf \left\{\left\|x-T^{*} y\right\|_{1}^{2}+\frac{1}{n}\|y\|_{\Sigma}^{2} ; y \in \sum_{\ell_{2}} C\left(K_{n}\right)^{*}\right\}, \\
\|\| x\left\|^{2}=\right\| x \|_{1}^{2}+\sum_{n=1}^{\infty} 2^{-n}|x|_{n}^{2} .
\end{gathered}
$$


This is a dual norm and we will prove that it is p-UR. Choose $x_{i}, y_{i} \in l_{1}(K)$ such that $\left\|x_{i}\right\|_{1} \leq 1,\left\|y_{i}\right\|_{1} \leq 1$ and

$$
\lim _{i \rightarrow \infty}\left(\left.2\left\|x_{i}\right\|\right|^{2}+2\|\| y_{i}\|\|^{2}-\left\|\mid x_{i}+y_{i}\right\|^{2}\right)=0 .
$$

Then for all $n \in \mathbb{N}$,

$$
\lim _{i \rightarrow \infty}\left(2\left|x_{i}\right|_{n}^{2}+2\left|y_{i}\right|_{n}^{2}-\left|x_{i}+y_{i}\right|_{n}^{2}\right)=0 .
$$

The infimum in the definition of $|\cdot|_{n}$ is attained (see [5, p. 44]); e.g., for all $i, n \in \mathbb{N}$ there are $u_{i}^{(n)}, v_{i}^{(n)} \in \sum_{\ell_{2}} C\left(K_{n}\right)^{*}$ such that

$$
\begin{aligned}
\left|x_{i}\right|_{n}^{2} & =\left\|x_{i}-T^{*} u_{i}^{(n)}\right\|_{1}^{2}+\frac{1}{n}\left\|u_{i}^{(n)}\right\|_{\Sigma}^{2} \\
\left|y_{i}\right|_{n}^{2} & =\left\|y_{i}-T^{*} v_{i}^{(n)}\right\|_{1}^{2}+\frac{1}{n}\left\|v_{i}^{(n)}\right\|_{\Sigma}^{2} .
\end{aligned}
$$

From (2) we get

$$
\left\|u_{i}^{(n)}\right\|_{\Sigma} \leq n\left|x_{i}\right|_{n} \leq n\left\|x_{i}\right\|_{1} \leq n,
$$

and by the same manner we have $\left\|v_{i}^{(n)}\right\|_{\Sigma} \leq n$; therefore, for all $k \in K$ and $l \in N(k)$

$$
\left(u_{i}^{(n)}-v_{i}^{(n)}\right)\left(\widetilde{k}_{l}\right) \leq\left\|u_{i}^{(n)}-v_{i}^{(n)}\right\|_{1} \leq c .\left\|u_{i}^{(n)}-v_{i}^{(n)}\right\|_{\Sigma} \leq 2 c n,
$$

where $c$ is a constant of the equivalence of norms $\|\cdot\|_{1}$ and $\|\cdot\|_{\Sigma}$. From (1), (2), (3) we have

$$
\lim _{i \rightarrow \infty}\left(2\left\|u_{i}^{(n)}\right\|_{\Sigma}^{2}+2\left\|v_{i}^{(n)}\right\|_{\Sigma}^{2}-\left\|u_{i}^{(n)}+v_{i}^{(n)}\right\|_{\Sigma}^{2}\right)=0 .
$$

The norm $\|\cdot\|_{\Sigma}$ is p-UR and hence for all $k \in K, m \in \mathbb{N}$ and $n \in N(k)$

$$
\lim _{i \rightarrow \infty}\left(u_{i}^{(m)}-v_{i}^{(m)}\right)\left(\widetilde{k}_{n}\right)=0 .
$$

We can assume (by passing to a subsequence), that $\lim _{i \rightarrow \infty}\left|x_{i}\right|_{n}=d_{n}$. For every $x \in l_{1}(K),|x|_{n}$ is a nonincreasing sequence, hence there is $d=\lim _{n \rightarrow \infty} d_{n}$. By passing to a subsequence again, we can assume, moreover, that $\lim _{i \rightarrow \infty}\left|y_{i}\right|_{n}=d_{n}$. Choose $\varepsilon>0$ and $k \in K$. Put $A=K \backslash\{k\}$. Let $m \in \mathbb{N}$ be such that $d_{m}<d+\varepsilon$. Then

$$
\left|\left(x_{i}-y_{i}\right)(k)\right| \leq\left|\left(x_{i}-T^{*} u_{i}^{(m)}\right)(k)\right|+\left|\left(T^{*} u_{i}^{(m)}-T^{*} v_{i}^{(m)}\right)(k)\right|+\left|\left(T^{*} v_{i}^{(m)}-y_{i}\right)(k)\right| .
$$

Considering the second term, we have

$$
\begin{aligned}
\left|T^{*}\left(u_{i}^{(m)}-v_{i}^{(m)}\right)(k)\right| & =\left|\sum_{n \in N(k)} \frac{1}{n^{2}}\left(u_{i}^{(m)}-v_{i}^{(m)}\right)\left(\widetilde{k}_{n}\right)\right| \\
& \leq \sum_{n \leq n_{0}, n \in N(k)}\left|\frac{1}{n^{2}}\left(u_{i}^{(m)}-v_{i}^{(m)}\right)\left(\widetilde{k}_{n}\right)\right|+\varepsilon,
\end{aligned}
$$

where $n_{0}$ depends only on $\varepsilon$ (because of (4)) and the sum is finite and therefore tends to 0 for $i \rightarrow \infty$ because of (5). It remains to prove, that $\left|\left(x_{i}-T^{*} u_{i}^{(m)}\right)(k)\right|<\varepsilon$. We can assume that $k \in K_{n_{0}}$. Put $y=s_{i}+\left(\left.u_{i}^{(m)}\right|_{\tilde{A}}\right)$, where $s_{i}(l)=n_{0}^{2} x_{i}(k)$ if $l=\tilde{k}_{n_{0}}$, 
and $s_{i}(l)=0$ otherwise. Considering this $y$ in the definition of $\left|x_{i}\right|_{n}$ we get

$$
\begin{aligned}
\left|x_{i}\right|_{n}^{2} & \leq\left\|\left.\left(x_{i}-T^{*} u_{i}^{(m)}\right)\right|_{A}\right\|_{1}^{2}+\frac{1}{n}\left\|s_{i}+\left(\left.u_{i}^{(m)}\right|_{\tilde{A}}\right)\right\|_{\Sigma}^{2} \\
& \leq\left\|\left.\left(x_{i}-T^{*} u_{i}^{(m)}\right)\right|_{A}\right\|_{1}^{2}+\frac{1}{n}\left(\left\|s_{i}\right\|_{\Sigma}+\left\|\left.u_{i}^{(m)}\right|_{\tilde{A}}\right\|_{\Sigma}\right)^{2}, \\
& \leq\left\|\left.\left(x_{i}-T^{*} u_{i}^{(m)}\right)\right|_{A}\right\|_{1}^{2}+\frac{1}{n}\left(n_{0}^{2} c+m c^{2}\right)^{2}
\end{aligned}
$$

because

$$
\left\|\left.u_{i}^{(m)}\right|_{\widetilde{A}}\right\|_{\Sigma} \leq c\left\|\left.u_{i}^{(m)}\right|_{\widetilde{A}}\right\|_{1} \leq c\left\|u_{i}^{(m)}\right\|_{1} \leq m c^{2},
$$

where we used that the canonical norm $\|\cdot\|_{1}$ on $X^{*}$ is a lattice norm.

Hence for all $n \in \mathbb{N}$

$$
\limsup _{i \rightarrow \infty}\left\|\left.\left(x_{i}-T^{*} u_{i}^{(m)}\right)\right|_{A}\right\|_{1}^{2} \geq \lim _{i \rightarrow \infty}\left|x_{i}\right|_{n}^{2}-\frac{1}{n}\left(c n_{0}^{2}+m c^{2}\right)^{2} .
$$

Finally,

$$
\limsup _{i \rightarrow \infty}\left\|\left.\left(x_{i}-T^{*} u_{i}^{(m)}\right)\right|_{A}\right\|_{1}^{2} \geq d^{2}
$$

For all $i \in \mathbb{N}$ we have

$$
\begin{aligned}
\left|\left(x_{i}-T^{*} u_{i}^{(m)}\right)(k)\right| & =\left\|x_{i}-T^{*} u_{i}^{(m)}\right\|_{1}-\left\|\left.\left(x_{i}-T^{*} u_{i}^{(m)}\right)\right|_{A}\right\|_{1} \\
& \leq\left|x_{i}\right|_{m}-\left\|\left.\left(x_{i}-T^{*} u_{i}^{(m)}\right)\right|_{A}\right\| .
\end{aligned}
$$

Hence we get

$$
\liminf _{i \rightarrow \infty}\left|\left(x_{i}-T^{*} u_{i}^{(m)}\right)(k)\right| \leq d_{m}-d \leq \varepsilon .
$$

The same holds for the third term and this concludes the proof.

Theorem 5. Let $K$ be a scattered Eberlein compact space. Then $C(K)^{*}$ admits an equivalent dual norm that is $L U R$ and $p$-UR with $\mathbb{F}=\left\{e_{k} ; k \in K\right\}$. In particular, $C(K)^{*}$ admits an equivalent dual norm that is LUR and URED.

Proof. K. Alster proved in [1] that if $K$ is a scattered Eberlein compact space, then $K$ is a strong Eberlein compact space, e.g., $K \subset\{0,1\}^{\Gamma}$ for some $\Gamma$. Hence

$$
K=\bigcup_{n=1}^{\infty} K_{n}, \text { where } K_{n}=\{x \in K ; \operatorname{card}(\{\gamma \in \Gamma ; x(\gamma)=1\}) \leq n\} .
$$

The $K_{n}$ 's are uniform Eberlein compact spaces, they are scattered and $K^{(n+1)}=\emptyset$. Hence by Corollary 2, $C\left(K_{n}\right)^{*}$ admits an equivalent dual norm that is both p-UR with $\mathbb{F}=\left\{e_{k} ; k \in K_{n}\right\}$ and LUR. Thus we can use the preceding theorem to finish the proof.

\section{Open QUESTION}

It is shown in 12, Th. 1] that if $X$ has an unconditional Schauder basis and $X^{*}$ admits an equivalent URED norm, then $X^{*}$ admits an equivalent dual weak* uniformly rotund norm. Because there is a scattered Eberlein compact space $K$ that is not uniform Eberlein compact (see, e.g., 2, Example 1.10]), the space $C(K)^{*}$ admits an equivalent dual p-UR (and hence URED norm) but does not admit any equivalent dual $\mathrm{W}^{*} \mathrm{UR}$ norm. But we do not know the answer to the following questions. Is there any reflexive Banach space $X$ such that $X$ admits an equivalent URED norm and does not admit any equivalent p-UR (and hence $\mathrm{W}^{*} \mathrm{UR}$ ) norm? 
Is there any Banach space that admits an equivalent URED norm and does not admit any p-UR norm?

\section{REFERENCES}

[1] K. Alster, Some remarks on Eberlein compacts, Fundamenta Mathematicae 104 (1979), 4346. MR 80k:54035

[2] S. Argyros and V. Farmaki, On the structure of weakly compact subsets of Hilbert spaces and applications to the geometry of Banach spaces, Trans. Amer. Math. Soc. 289 (1985), 409-427. MR 86h:46023

[3] Y. Benyamini, M. E. Rudin and M. Wage, Continuous images of weakly compact subsets of Banach spaces, Pacific J. Math. 70 (1977), 309-324. MR 58:30065

[4] J.M.F Castillo and M. González, Three space problems in Banach space theory, Lecture Notes in Math., Springer-Verlag, 1997. MR 99a:46034

[5] R. Deville, G. Godefroy and V. Zizler, Smoothness and Renormings in Banach Spaces, Monographs and Surveys in Pure and Applied Mathematics 64, Pitman, 1993. MR 94d:46012

[6] M. Fabian, G. Godefroy and V. Zizler, The structure of uniformly Gâteaux smooth Banach spaces, Israel J. Math. 124 (2001), 243-252. MR 2002g:46015

[7] M. Fabian, P. Habala, P. Hájek, V. Montesinos, J. Pelant and V. Zizler, Functional Analysis and infinite dimensional geometry, Canadian Math. Soc. Books, 8 (Springer-Verlag), 2001. MR 2002f: 46001

[8] W. B. Johnson and J. Lindenstrauss, Some remarks on weakly compactly generated Banach spaces, Israel J. Math. 17 (1974), 219-230. MR 54:5808

[9] A. Moltó, J. Orihuela and S. Troyanski, Locally uniformly rotund renorming and fragmentability, Proc. London Math. Soc. (3) 75 (1997), 619-640. MR 98e:46011

[10] S. Negrepontis, Banach Spaces and Topology, Handbook of Set-Theoretic Topology (K. Kunen and J. Vaughan, eds.), North-Holland, 1984, pp. 1045-1142. MR 86i:46018

[11] M. Raja, Mesurabilité de Borel et renormages dans les espaces de Banach, (Doctoral dissertation), Printemps, 1998.

[12] J. Rychtář, Uniformly Gâteaux differentiable norms in spaces with unconditional basis, Serdica Math. J. 26 (2000), 353-358. MR 2003a:46025

[13] D. Yost, The Johnson-Lindenstrauss Space, Extracta Mathematicae 12 (1997), 185-192. MR 99a:46027

[14] V. Zizler, Nonseparable Banach spaces, Handbook on Banach spaces (W. B. Johnson and J. Lindenstrauss, eds.), to appear.

Department of Mathematical Analysis, Charles University, Faculty of Mathematics and Physics, Sokolovká 83, 18675 Praha 8, Czech Republic

E-mail address: rychtar@karlin.mff.cuni.cz

Current address: Department of Mathematics and Statistics, University of Alberta, Edmonton, Alberta, Canada T6G 2G1

E-mail address: jrychtar@math.ualberta.ca 